\title{
Socio-technical analysis of IPTV: a case study of Korean IPTV
}

\author{
Dong H. Shin
}

Dong H. Shin, Assistant

Professor, School of

Information Sciences and

Technology, Pennsylvania

State University at Berks

Campus, Reading,

Pennsylvania, USA
The author thanks Dr Murali Venkatesh for his valuable guidance on the socio-technical approach.

\begin{abstract}
Purpose - This paper seeks to investigate the prospects for internet protocol TV (IPTV) from the social, economic and regulatory perspective to gain a better understanding of how IPTV will evolve and stabilize in next generation network environments.

Design/methodology/approach - Qualitative and quantitative data were collected from multiple data collection methods. The analysis is based on theoretical frameworks derived from socio-technical theories and web of stakeholder analyses.

Findings - From the socio-technical analysis, the development of IPTV implies that IPTV has been through internally-driven change (more or less technology-driven and regulatory-constrained), but from now on it will likely be driven by external factors (market and user). The web of stakeholder analysis implies that the further development of IPTV in Korea is likely to comprise multiple stages, predicated by several significant events: the deployment of Broadband Convergence Network and FTTH, the resolving of content issues, establishing standards, and the introduction of new regulatory frameworks.
\end{abstract}

Practical implications - The case of Korea offers implications for other countries that are pursuing IPTV development strategies.

Originality/value - By looking at IPTV as a sociotechnical ensemble, this paper seeks a firm understanding of the interaction among IPTV technology, its market, industry, and regulation regarding IPTV. A stakeholder analysis helps this study to address such interactions.

Keywords Transmission Control Protocol/Internet Protocol, Television, Sociotechnical change, South Korea

Paper type Research paper

\section{Introduction}

Internet protocol TV (IPTV) is being rapidly developed around the world. IPTV is a new media technology capable of receiving and retrieving a video stream encoded as a series of internet protocol packets. IPTV is changing the notion of traditional broadcasting and telecommunications. The speedy trend of convergence has resulted in a new stream of personalized and highly interactive TV broadcasting services. Its versatility, which can provide high quality audio and video content, has been the most significant competitive advantage. In addition, IPTV's flexibility and scalability of terminals adds a significant edge. IPTV will become a common platform for systems where television and/or video signals are distributed to subscribers or viewers using a broadband connection over internet protocol. IPTV is expected to evolve into a universal service under a ubiquitous computing environment and have an immense influence over other industries engaged in terminal and part production or content development. Considering all of these projected impacts, IPTV has a catalytic power, opening a new technological generation, the next generation network environment.

Korea has been taking a leadership role in developing a series of convergence technologies from wireless broadband, digital multimedia broadcasting (DMB), and IPTV. With the high broadband penetration, the prospect of IPTV seems to be far brighter, which makes experts wonder how far ahead the South Koreans are with respect to IPTV. With the launching of DMB 
in Korea, telecommunications and IT firms are taking the next step to prepare the next generation of convergence service, IPTV. While IPTV is quickly moving from an experimental to a commercial stage, industries are questioning the technological feasibility and commercial marketability. Indeed, there is a fuzzy and hazy prospect about the impact and benefit of IPTV. It appears that IPTV will provide an edge to telecommunications operators in competing with cable and broadcasters. Yet, the question is how soon market will absorb the benefits IPTV has to offer or simply put, whether the technology is time to market. This question gains even more attention with the current DMB market share. Unlike its initial prediction, DMB in Korea has not drawn significant subscribers as of 2005. There is a concern that IPTV, as a variation of DMB, will be likely to follow its predecessor. The increased uncertainty shifts our attention from technology-push perspective to demand-pull perspective; the forces driving the development of convergent services; whether the forces come from market demand side or industry supply side; and, whether there are enough market demands to justify continued development. Eventually, such dichotomy of push and pull perspective will be displaced with a comprehensive and holistic framework for the next generation of network environments.

This study addresses these questions in order to identify prospectus, limitation and uncertainty embedded in the development IPTV in Korea. By identifying possible destructive powers, this study finds a strategic option of IPTV among appropriate regulation, industry dynamics, and market receptiveness. To this end, a socio-technical approach (Bijker, 1995; MacKenzie and Wajcman, 1999; Sawyer et al., 2003) is used to investigate the complex and underlying issues of IPTV. In addition, this paper uses stakeholder analysis (Mitroff, 1983; Freeman, 1984; Papazafeiropoulou and Pouloudi, 2000) to focus on the impact of various strategies on stakeholders. This relationship, between technology and society, between technical artifacts and the discourse that surrounds it, is an essential insight into the way technologies like IPTV have been designed, deployed, and discussed. Despite optimistic projects, uncertainty still remains about where IPTV will evolve and how it will impact the new socio-technical milieu. The aim is to provide another perspective for the understanding of the convergence technology diffusion process in an exemplary country. By doing so, recommendations for the development of convergence technologies strategies can be offered.

\section{Socio-technical approach to IPTV}

A socio-technical perspective can provide a stronger framework than any other approaches because of its integrative and holistic nature (MacKenzie and Wajcman, 1999). Using a socio-technical framework, Sawyer et al. (2003) investigate emerging broadband and mobile opportunities with the socio-technical perspective. By emphasizing the complex relations between social and technical aspects of a technology, Sawyer et al.'s (2003) study shows how a socio-technical approach can be used in predicting emerging technologies to provide useful insight into the emerging technologies. Sawyer et al. (2003) described the technological forces (technology advancement) and the social forces (regulation/government/market/users) of broadband diffusion and the interactions of the two.

By using the socio-technical perspective, this study makes a contribution to theoretical advancement. Although a socio-technical perspective has been primarily used in designing organizational systems considering social and technical factors (Orlikowski, 2002; Sawyer, 2001), it has been rarely used in emerging technologies at macro levels considering various relations (industry, regulation, and society). With the three entities of socio-technical framework, this study focuses on relations among the three entities in order to capture the dynamics of technological change and in order to link the social interaction and technological choices. In the end, by looking at IPTV as a sociotechnical ensemble, this paper seeks a firm understanding of the interaction among IPTV technology, its market, industry, and regulation regarding IPTV.

\section{A web of stakeholder: diffusion of technology innovation}

As an enhancing tool to focus on the relations among the entities of the socio-technical framework, this study complements the diffusion literature to the socio-technical framework. 
Commonly, a socio-technical system theory has been criticized neglecting interaction among entities (Winner, 1997; MacKenzie and Wajcman, 1999; Williams and Edge, 1996). To overcome this weakness, this study complements the socio-technical framework with three layers of diffusion process. Applying Damsgaard's (1996) three layers of the diffusion processes (organizational, industry, and environment layers), Choudrie and Lee (2003) used the three layers of diffusion process to investigate the broadband diffusion factor in Korea.

The organizational layer consists of individual users and organizational entities using the IT. The industry layer consists of organizations and institutions sharing a stake in the same function, market area or part of the value system. The environmental layer is divided into two components: the institutional layer and the regulatory layer. The institutional layer consists of entities such as international agencies, trade associations and higher education institutions. The regulatory layer is related to the telecommunications, business and privacy regulations applied by government regimes in order to set the normative boundaries for interactions between the trading partners. One approach to examining the environmental layer of IT diffusion was made by King (1994) in their study on understanding the role of the government and other institutions in IT innovation. They observed that the mechanisms used for the mobilization of government leadership appear to be inefficient although the objectives of IT-related programmatic statements issued by various government agents are clear. They argued that difficulties in the application of IT diffusion polices are related to inefficient analysis of the role of institutions involved in the IT diffusion process.

The institutional layer is mostly related to interactions of the stakeholders involved in the IT diffusion process (Damsgaard, 1996). These interactions have an influential rather than regulatory character: the institutional layer corresponds to influential actions. Similarly the regulatory layer deals with the application of laws and restrictions by government regimes and is related to regulatory actions. Building on this, Papazafeiropoulou and Pouloudi (2000) used stakeholder analysis (Mitroff, 1983; Freeman, 1984) for further investigating the institutional layer of innovation diffusion in the case of electronic commerce. The stakeholder approach can contribute to an understanding of the institutional layer of the innovation diffusion process by examining stakeholders' roles, interests and hidden agendas. Papazafeiropoulou and Pouloudi (2000) focused on the role of the government and argued that governments should have a holistic view of the stakeholders operating in the market and act pro-actively in a rapidly technologically changing environment in order to be effective in the application of their strategies. They identified five groups of stakeholders: the national government, international organizations, policy intermediaries, companies, and consumers/citizens.

In this paper the three layers of diffusion with a reference to web of stakeholders are used to describe the diffusion strategies that the various stakeholders implemented and to show how these strategies were used in relation to other stakeholder groups.

\section{Methodology}

A variety of data collection methods were used in developing the case study. Qualitative and quantitative data were collected from multiple data collection methods - surveys, in-depth interviews, telephone interviews and e-mail exchanges - with people associated directly with the development of IPTV from industry players, consumer groups, regulators, consumer groups, academics, and researchers.

For the qualitative data, the interviewees were thoughtfully selected and a total of 19 in-depth interviews were conducted over a seven-month period (August 2005-December 2005). Due to constraints such as the scope and timeframe of this research, a sample of key stakeholders and participants, identified from background research of the case, were selected. All interviews used a combination of structured and unstructured stimulus items. Survey questionnaire were sent to those who could not schedule interviews, such as high level strategic positions and officials in industry and government. A total of 98 questionnaires were mailed out and 31 of those were returned. In addition to interviews, archival material, such as industry reports, government publications, technical reports, and any materials pertaining to the IPTV development, was collected and analyzed. Such archival materials 
were useful in obtaining factual data such as figures and statistics of IPTV subscribers. Using multiple sources of evidence increased the reliability and validity of the data in this study. Multiple sources of evidence increased confidence in findings, strengthened the validity of findings, and reduced methodological bias.

In addition to qualitative data, quantitative data were gathered and analyzed to assess users' attitudes toward IPTV. National surveys were administered by telephone during September 2005-December 2005. Individuals' responses to questions about whether they accept IPTV are collected and are combined with observations of their socio-economic status and intrinsic/extrinsic factors. Descriptive data results were analyzed by SPSS and factor analysis and logistic regression were used. The combination of quantitative and qualitative data has helped to enhance analysis and promote the validity of result, providing a more rounded understanding of the nature, scale and prediction of technology development. The value of using both quantitative and qualitative data is argued by Barton et al. (2002).

\section{IPTV as a socio-technical artifact}

The core assumption of a socio-technical approach is that a technology is not technology itself, but a socio-technical ensemble. With a socio-technical approach, this study investigates IPTV regulation, market, service, and users.

\section{Regulation: regulatory challenges surrounding IPTV in Korea}

The IPTV in Korea has faced regulatory setbacks in its early development. Because of the absence of a clear concept of convergence in relevant policy and regulation and the convergence service, IPTV in Korea has faced overlapping regulation in one case and non-regulation in other cases.

The Ministry of Information and Communication (MIC) and the Korea Broadcasting Commission (KBC) have each been attempting to take the initiative on IPTV, which has created a challenging political environment. The MIC regards IPTV as the offspring of the country's advanced telecommunications business and contends the convergence service should be under its control. In response, the KBC counters IPTV belongs to broadcasting and cable TV operators look to start IPTV services on their own. Among such confusion, the current classification of IPTV service has not been decided yet. So far IPTV is regarded as neither telecommunications nor broadcasting. Under the scheme, IPTV service requires both the amendment of current laws and establishment of new laws to appropriate laws, because it has difficulties regarding the number of channels that can fit on the whole telecom spectrum due to frequency limitations. The current Broadcasting Law has provisioned maximum share limitations in IPTV and has limited participation in IPTV service in order to keep the telecommunication operators from the broadcasting industry.

In addition to the battle between the regulators, structural problems pose more challenging obstacles. The current regulatory framework for Korean broadcasting and telecommunications services markets is categorized along with transmission media and divided into basic telecommunications, value-added telecommunications and special telecommunications services categories and terrestrial, cable, satellite broadcasting services in broadcasting services sectors respectively. Yet, such vertical division of service markets is no longer appropriate to properly regulate the converging market and services and has the risk of over-regulation against service providers. The regulatory structure in Korea seems to limit the wide possibility of convergences. The regulatory principles of broadcasting are stricter than those of telecommunication, and telecommunications and the IT industry have regulatory traditions different from those of broadcasting. If convergence services are regulated by the broadcasting regulatory framework, the convergence market will face strict entrance barriers and content regulation.

Until recently, the government role much represented by the KBC has been limited to preserving the public interest through universal service, preserving the diversity of communicators, and regulating public news reporting (Shin, 2004). The undermining question is about ontological and semantic distinction: Does IPTV belong to the 
telecommunication industry or is it a functional extension of broadcasting? The current practice of the KBC is to define IPTV as an extension of traditional broadcasting, based on the emerging medium's functionality. This places it within the framework of traditional broadcasting and, according to this framework, the KBC requires IPTV carriers to observe key broadcasting principles and public interests such as universal service. It is necessary to consider diversity issues in broadcasting services, which inherently observe principles of free speech. Along with the KBC, the incumbent broadcasters stand in firm opposition to retransmitting their content over IPTV in the name of broadcasting facilities as public resources given the magnitude of its impact on the public. The current battle between the broadcasting and telecommunications sector will be continued, and this battle will significantly undermine the development of convergence services.

Korea's telecommunications companies have technologies to start the IPTV but should wait until the MIC and the KBC straighten things up. Hanaro, the country's runner-up fixed-line operator, initially aimed to start IPTV services in 2005, but the timeline has been delayed indefinitely.

\section{Market}

The Korean information communication technology (ICT) market is very favorable to the development of IPTV. With 27.3 million users at the end of 2004, Korea represents the world's fifth largest internet market (OECD, 2004). Its internet penetration rate, 55.2 percent at end of 2004, makes it the third highest in the world (OECD, 2004). In addition, the growth in broadband access will be a significant factor in IPTV. Since the launch of broadband internet access in July 1998, the number of subscribers had risen to ten million by the end of 2002. Most services that needed broadband access, such as audio and video streaming, gaming and e-commerce, were only available on the internet so this created many new internet users. Currently, 79 percent of internet users log in via a broadband connection (OECD, 2004). Korea is closely crossed by several nationwide, gigabit fiber-optic backbones operated by telecommunication operators, internet providers and the electric company. International connectivity has been growing rapidly over the last several years. In addition, the Korean government's new infrastructure project, Broadband Convergence Network, will be a perfect platform for IPTV. The Korean Government has pursed the Broadband Convergence Network $(\mathrm{BcN})$ project with the vision of providing the core infrastructure for Broadband IT Korea by establishing the world's first BcN. The infrastructure for the 20 million subscribers to enjoy continuous multimedia service in $50 \sim 100 \mathrm{Mbps}$ bandwidth will be built by 2010. The target is for BcN-related broadcasting and communication-related equipment manufacturing.

\section{Users}

While these situations will be certainly beneficial factors to the development of IPTV, the question is, how much customer demand will be in market and how will users react to IPTV. This section describes the qualitative assessment and quantitative data analysis of Korean users to better predict the market and users of IPTV.

\section{Qualitative assessment of the Korean consumers - special features of Korean digital consumers}

Korean consumers have traditionally shown a very high rate of acceptance of new technology and services, as is apparent in the world's highest rates of internet and wireless service usage. Han (2003) found that Koreans have very sophisticated consumer tastes and preferences. It is well known that Korean consumers are choosy, and their demands are increasingly reflected in new products. Sales of digital cameras, for example, are increasing with double-digit growth rates, demonstrating why major global level companies are targeting Korean consumers so enthusiastically. Indeed, the Korean digital consumers' behavioral patters are those of early adopters of technology, which is crucial when it comes to penetration and adoption of new media (Shim, 2005a). For example, when new electronics products and mobile phones are first released and marketed to the public, Koreans race to buy new tech gadgets ahead of others. There are many studies 
characterizing Korean users as early adopters. One of the factors ascribed to the early adopters is a high education. Education is a high priority in contemporary Korean society. Parents are very committed to providing the best education possible for their children and spend a significant proportion of their disposable income for educational purposes, such as extracurricular tutoring. This strong emphasis on education and academic performance has prompted parents to turn to the internet for educational goods and services. Both the government and commercial players deliberately promoted the educational benefits of IP-based services and used them as a means for driving demand.

Second, strong interests in value-added service characterize Korean consumers. The growing strength of the online gaming market meant that children and teenagers had their own strong motivations - beyond their educational requirements - for encouraging their parents to provide them with broadband access at home. Anecdotal evidence suggests that, when asked, Koreans are proud of explaining the benefits and value they gain from broadband. To them the benefits are clear and obvious. Users almost always refer to education and entertainment. Other major benefits include access to information, financial transactions and viewing time-shifted television content. Culturally, Korean digital revolution is a reflection of a hurry-up culture (Shim, 2005a). Koreans live in an intense, speed-up culture where all processes are speeded up. This view applies to a wide range of behavioral patterns, from eating rapidly to acquiring the latest model of mobile phones.

Third, the housing pattern is another favorable factor that will accelerate Korean IPTV adoption by substantially reducing the deployment cost in densely populated areas, and by providing opportunities for the multi-dwelling unit residents to compare what other neighbors have at their homes. More than half of Korea's 48 million population live in an apartment complex and now some 50,000 households are living in virtual apartments characterized by built in broadband lines, home automation and online neighborhood systems. Local builders are scrambling to wire homes to provide state-of-art living environments fit for busy, digitally advanced Koreans. As a result of this special housing pattern, Korea has one of the best broadband infrastructures in the world, and now they are the largest in broadband market penetration. They already have about 10-15 million broadband users and 40-50 percent of households. The widespread broadband infrastructure will work favorably to the development of IPTV.

\section{Quantitative predication of customers' preferences}

From quantitative survey, this study sought to answer two questions: How users will respond to IPTV and what are customers expecting from IPTV? This study acquired quantitative data from the Korean Ministry of Information and Communications who generously provided this study with a pool of respondents. Then, this study outsourced the telephone interviews on the pool of respondents. A private research company carried out extensive telephone surveys among a randomly selected 412 people from August 2005 to October 2005. The survey questionnaire asked people whether they would adopt IPTV with step-wise factors. Through mail survey, e-mail, and online survey, 40 valid responses were collected. For analysis of a basic statistics and analysis of logit and variance, SPSS 10.0 is used.

For the internal consistency and the reliability of the data, factor analysis was performed to reduce the number of variables and to avoid multi-collinearity. A principal components method with a varimax rotation of the 13 variables reveals six underlying factors with eigenvalue (characteristic value) of greater than one. These six factors explained 75.9 percent of the variability in the 13 variables. A description of each factor and its corresponding variables are:

- intrinsic factors;

- extrinsic factors;

- economic factors; and

- demographics. 
A confirmatory factor analysis was performed on the data to analyze the constructs' validity on units remaining after discarding items disturbing the homogeneity of the set. The confirmatory factor analysis indicated that the factors generally satisfied validity evaluation standards.

After the factor analysis, multivariate analysis was performed to arrive at a logistic regression model, which enables this study to estimate the relative importance of each predictor. The dependent variable has only two values ( $1=$ adopt, $0=$ not adopt), thus logistic regression analysis is the most appropriate type of analysis. A logit regression was performed for each of the factors. A logit model helps to understand the extent to which such factors as intrinsic or extrinsic factors influence a customer's choice of product. It evaluates the influence of independent variables on an event either happening or not happening; either accepting IPTV or not. In the analysis of the independent variables (continuous, dichotomous, and categorical) in this study, each of the categories is assessed for its impact on the dependent variable (accept or not accept IPTV). A series of people are questioned as to whether or not they would accept IPTV. For each respondent, the variables of content, service, and compatibility and subscribing $(1=$ yes, $0=$ no) are recorded.

A questionnaire was designed based on previous studies that identified diffusion factors of DMB. The questionnaire's items are made up based on these study variables and it is evaluated by a pilot test done by relevant experts and company executives. The LOGIT procedure is used to fit a logistic regression model to the probability of a positive response (yes) as a function of the variable of sex and age. Specifically, the probability of subscribing is modeled as:

$$
\begin{aligned}
p & =\operatorname{Pr}(\text { accept }=1) \\
& =F\left(b_{0}+b_{n}[\text { intrinsic factors }+ \text { extrinsic factors }+ \text { demographics }+ \text { economics }]+\alpha ́\right)
\end{aligned}
$$

where $F$ is the cumulative logistic distribution function and $p$ is the probability of adoption of IPTV given $X$ (variables). $P$ can be calculated as:

$$
\begin{aligned}
& P_{a}(1 \mid x)=\frac{\operatorname{EXP}\left(U_{a}\right)}{1+\operatorname{EXP}\left(U_{a}\right)} \\
& U_{a}=b_{0}+b_{1} X_{1}+b_{2} X_{2}+\ldots+b_{n} X_{n}+e .
\end{aligned}
$$

$U_{a}$ is the utility function of event a expressed as a linear combination of a number of explanatory variables $X_{1}, X_{2}, \ldots$, and $b_{n}$ is the estimated parameter of variable $X_{n}$. A greater value of $U_{a}$ implies a greater probability for the event to take place. When $U_{a}$ approaches infinity, $P_{a}$ approaches 1 , indicating a high likelihood for the adoption of IPTV to occur. When $U_{a}$ approaches negative infinity, $P_{a}$ approaches 0 . When $U_{a}$ equals zero, the probability is 0.50 , implying a 50/50 chance for the adoption of IPTV to occur. Although logistic regression does not produce an $R^{2}$, which is equivalent to the $R^{2}$ in linear regression, many studies have shown the validity using the pseudo- $R^{2}$ (Menard, 1995). As the $R^{2}$ is an important measure of predictive power, the pseudo- $R^{2}$ is employed in the analyses presented here.

From the result of surveys, the two most important variables in explaining the users' choices are content and services (Table I). Such variables can be more clearly conceptualized in terms of intrinsic and extrinsic factors; intrinsic desire to enjoy individualized services and content, and extrinsic desire to interact with others. Users seem to expect increased control and augmented interactivity from IPTV. Through IPTV, users seem to want to skip advertising, freely choose their content, and who can manage various interactive/proactive services (evolving from passive viewers to active producers). Users appear to want the ability to easily pick and mix interactivity-rich content and additional services beyond just current video on demand. Operational implications and industrial strategies of IPTV can be derived from the variables identified in this study. Convergence technologies blur the lines, but users see the technologies differently and thus business models will be completely different. What users expect from IPTV may be a new paradigm, not just a television box change. This suggests that IPTV providers follow Blue Ocean Strategy to develop IPTV as a totally 
Table I

\begin{tabular}{|c|c|c|c|c|}
\hline \multirow[b]{2}{*}{ Factors } & \multirow[b]{2}{*}{ Independent variables } & \multicolumn{3}{|c|}{ IPTV adoption } \\
\hline & & $B$ & $S E$ & $p$-value \\
\hline \multirow[t]{3}{*}{ Intrinsic factors } & Timely/on-demand & 0.404 & 0.568 & 0.002 \\
\hline & Special functionality & $0.478^{\star *}$ & 1.731 & 0.007 \\
\hline & Individualized content & $0.743^{\star \star}$ & 0.444 & 0.0183 \\
\hline \multirow[t]{3}{*}{ Extrinsic factors } & Interactivity & $0.550^{\star *}$ & 3.215 & 0.031 \\
\hline & Value-added service & $1.019^{* \star}$ & 1.122 & 0.849 \\
\hline & Compatibility & $1.080^{\star \star}$ & 0.0164 & 0.364 \\
\hline \multirow[t]{3}{*}{ Cost } & Fixed cost & $2.124^{*}$ & 0.012 & 0.049 \\
\hline & Monthly fee & 1.003 & 1.323 & 0.783 \\
\hline & Additional service charge & 0.923 & 1.822 & 0.503 \\
\hline \multirow[t]{3}{*}{ Demographics } & Experience & $1.392^{* *}$ & 0.923 & 0.001 \\
\hline & Age group & $2.394^{\star *}$ & 1.394 & 0.004 \\
\hline & Income & 3.922 & 3.234 & 0.873 \\
\hline & $0.000^{\star *}$ & -4.977 & 1.456 \\
\hline \multicolumn{2}{|l|}{ Log likelihood } & 164.788 & & \\
\hline \multicolumn{2}{|l|}{ PCE } & 87 & & \\
\hline \multirow[t]{2}{*}{ Chi-square } & & 3.84 & & \\
\hline & & For alpha $=0.05^{\mathrm{a}}$ & & \\
\hline
\end{tabular}

different service that makes the competition irrelevant from other competitors like cable, satellite, and telcos.

The customers' preference to interactivity implies that users desire a higher level of interactivity, for example, proactive levels; users not only seek interactive nature of services and functions, but they also want to have direct relation with program providers or carriers and want to selectively access and control content as much as possible. The interactively offered through the wide open web-based environment of the internet has enabled users to have control over a vast amount of content. In response to this, the future IPTV system will be effective with an end-to-end system that can eliminate or minimize the layers of middlemen like advertisers. This end-to-end approach can be effective in gaining complete control of the customers through a closed, proprietary system, which Microsoft is currently developing.

The significance of service and content variable implies that providers should offer effectively different services and content from those of traditional carriers. The pay-TV market has been reaching saturation, and, therefore, IPTV providers will need to capture market share through effective differentiation. Compelling content packaging, on-demand, interactive applications, and, ultimately, integration of video and video devices into the digital home will be the key factors for IPTV. As to content, IPTV providers should offer a quality of content to remain competitive because local competition is driving them to move more toward personalization of choice and unique triple-play offerings. The importance of the content factor sheds light on tough negotiation with regulators and broadcasters to share broadcasters' content. Content partnerships with broadcasters, programmers, and service operators will make a big difference in IPTV, a dispute which happened during the development of DMB in Korea. Not only can customers bypass the cable and telephone programming networks, but also IPTV providers will be using the high speed portions of these networks to bypass their own broadcast networks. This fear may cause broadcasters to ask their governments for regulatory protection until they can get their own IPTV systems up and running efficiently. The current regulatory environment in Korea for television systems has been structured for the broadcast television industry, and these regulations may prevent or limit the authorization of IPTV providers to offer some types of innovative services. The question of how telecom carriers can bypass regulatory barriers to offer IPTV service will be interesting. While regulation may restrict domestic competition, regulators cannot block international companies from providing content over IP. In fact, international companies will be more effectively able to compete against existing incumbent broadcasters because users will rely on IP for content they cannot find through traditional media. 
As the diverse services will be emerging, compatibility is found to be an important variable that consumers consider with IPTV. Thus, a higher level of compatibility can be the critical factor to differentiate IPTV from other services. As users will seek a higher level of compatibility, compatibility issues with IPTV system will continue to appear over its diffusion. IPTV providers may have to answer how services can be bundled, what video-on-demand and interactive services networks will offer and how these services can deliver 1-1 relationship with the network operator. In particular, creating on-demand service bundles with phone, broadband, web access and other services will be a key success factor as shown in the triple play. The effort to increase compatibility will contribute to building multi-platform environment, creating economies of scale, promoting the deployment of a wider range of services, ultimately leading to lower prices for users.

In short, the findings of consumers' demand imply that IPTV involves the feature of demand pull as much as supply push. Users want IPTV and the market becomes more and more receptive to IPTV. It is highly probable that a large number of users will accept IPTV. Korean users tend to be technology-savvy and this trend appears to continue in IPTV. With the successful demand-nurturing strategies by government and industry, Korean users are acclimated to new emerging technologies. Unlike DMB, which seems not to be ready for killer application, IPTV has more chances to be developed mass market than DMB considering the infrastructure and its technical issues are better integrated into social context. The identified dimensions of intrinsic and extrinsic factors are falling into the new business models that telecom carriers are trying to develop. Sooner or later, IPTV providers will develop multiplatform to content and service delivery which will have to be fully interoperable and compatible with those provided and created by other sources.

\section{A web of stakeholders and strategies in the development of IPTV in Korea}

In the analysis using web of stakeholders, three relations are analyzed:

1. government vs market;

2. government vs industry; and

3. interactions among industry players.

The web of stakeholder analyses show that the true meaning and potential of IPTV are largely misunderstood - and not just by the regulators, but by many within IT industry as well.

\section{Government vs market: environment layer}

Although the government's main influence has been in promoting the shared vision, the government has intervened more directly to create market environment that is highly receptive to new technologies. There are two types of interventions: intervention at the supply chain and intervention at the demand chain. The supply chain intervention has been designed to boost national connectivity at the regional level, in particular through the funding of the public internet backbone, the Korean Information Infrastructure, The Cyber 21 project, Digital Cities. This meant that the private sector could push through with the market-end of the supply chain, reaching a high percent household connectivity for IPTV.

Intervention at the market end of the demand chain, for instance by providing IT training for some ten million Koreans in occupations like government, the army, the teaching profession and homemakers, helps explain why Korea has been quick to exploit new ICT technologies: they create new domestic markets driving demand for new telecommunication services to be produced by local manufacturers.

Korea's distinction between the supply side and demand side is one of the factors of its success. It is equally important to note that the government has actively tried to create an information society. While the construction of an information infrastructure alone does not guarantee that people will actually make use of new ICTs, the provision of training and awareness campaigns helps people to join the information society. Thus, the government has addressed both the supply and the demand side of the equation. 
In 2004, Korean government initiated IT839 Strategy, a national information and telecommunication initiative, to spur the information society initiative. To effectively implement the IT839 strategy, the government has encouraged investment in developing eight new services such as wireless broadband (WiBro), satellite and terrestrial DMB, home network, telematics and radio frequency identification (RFID)-based services. The three major network infrastructures include the broadband convergence network, u-sensor network, and the next-generation internet protocol (IPv6). The broadband convergence network $(\mathrm{BCN})$ aims to prepare the nation for the era of convergence between telecommunications and broadcasting. The u-sensor network is being highlighted with the advent of the ubiquitous society. Next-generation internet protocol (IPv6) is expected to provide the basis for the Korean IT industry's future growth. The government's nine new growth engine sectors are next-generation mobile communications, digital TV broadcasting, home networks, IT system on chips (SoCs), next-generation PCs, embedded software, digital contents and software solutions, telematics, and intelligent service robots.

IT839 strategy has worked favorably to the development of IPTV. The IT839 strategy promotes the development of the IT industry that nurtures eight communication broadcasting services, three state-of-the-art infrastructures (networks), and nine IT new growth engines synthetically. The IT839 strategy is founded on the belief that IT will bring qualitative changes to the nation's economic growth paradigm. The IT industry is not only crucial for boosting the nation's exports, but also creating value in a knowledge-based economy. IPTV involves almost every component of IT839. IPTV will contribute to building a broadband convergence network and an IPv6 network and IPTV relates the eight new services, such as mobile communications, wireless broadband, RFID, and telematics. IPTV concerns the nine new growth engines, such as embedded software and intelligent robots. IPTV is indeed in the middle of IT839. The government wants to see IPTV as a test-bed of IT839.

Not only has the government helped to build the network infrastructure, they have also provided demand side stimulants such as internet literacy programs. The government pursued a variety of policy measures for creating internet demands as well as networks. One example was the Ten Million Internet Education program. The government set up the Ten Million People Internet Education project in June 2000 to provide internet education to 10 million people through a range of programs. This promotion activity contributed to the nationwide internet boom, with 3.4 million people including 1 million housewives being provided with basic internet skills by December 2000. This focus on the demand side contributed to the nationwide internet boom in Korea altogether with PC bangs, online games, online stock trading and so on (Kim, 2001). A range of consumer and business applications are driving the demand that is at the source of the bandwidth explosion for IPTV. For instance, NCSoft (a Korean software company) is already the largest independent online gaming company in the world. Its popular Lineage game has some two million users in Korea and is sold in four other countries.

In addition, the Korean government set aside funding of $\$ 100$ million for the development of next generation digital broadcast technology (named the SmaTV project) that includes IPTV, interactive technology and T-commerce (television commerce) technologies. Such funding will be expensed from the subsidy of the IPTV phone. For human resources development, MIC is setting up "Digital Broadcast Research Centers" in universities to educate and produce experts in the field. The Korean government has high hopes that investment in IPTV will give further impetus to the country's economic growth. The government predicts that multimedia broadcasts will create 160,000 jobs over the next ten years, with $\$ 13$ billion in product sales and added services.

\section{Government vs industry: institutional layer}

The Korean government's push and drive to meet huge consumer demand for digital ventures has allowed Korean Chaebols (associations of many firms clustered around a parent company) to grow as world digital leaders. Rather than discouraging this kind of apparent distorted industry structure, it tacitly approves it as a way to encourage more 
infrastructure deployments in a country that has the densest broadband infrastructure in the world. Korean Chaebols play a key role in IPTV infrastructure by using aggressive IT planning to develop IPTV. Over the past several decades, Korean Chaebols exerted enormous influence on IT and related sectors (Khanna and Palepu, 1999).

With the advent of IPTV, the South Korean government has been asked to modify its Chaebol-oriented structure. Because of the rising venture companies request and the nature of IPTV technology itself, the government considers adopting an open access policy approach to promote competition. In the past, the government's open access policy allowed any businesses that wanted to provide high-speed internet access to start and provide a variety of services following a simple registration procedure. This resulted in promoting high-speed internet access infrastructure and facilitated open competition in the high-speed internet market. The intense competition led to a low price and, subsequently, a rapid increase in demand. This open access policy seems to be applied in IPTV development.

As one business manager with a leading Korean company commented, "It is part of our business culture to listen to the government." The guidance provided by the Ministry of Information and Communication has been turned into action by the different companies active in the sector. This means that a little government spending can go a lot further in achieving objectives in Korea than in other countries where there is a more adversarial relationship between government and the private sector.

\section{Dynamics of industry players: organizational layer}

On the industrial supply side, IPTV has three products and service groups-content, transport, and processing. Several stakeholders are grouped in this category. First, telecom carriers (KT and Hanaro) are aggressively pursuing full-fledged IPTV services as a way of a new cash cow. ISPs and telcos see television as a potential extra application for the local networks, which they intend to build and unbundle. Second, cable TV service operators have fiercely opposed to IPTV, whose survival will be threatened when full-blown IPTV services start up. The third stakeholder is the terrestrial broadcasters who will offer the over-the-air content to the IPTV carriers.

\section{Cable vs telecommunications sector}

Two telecommunications operators are set to launch IPTV in 2006, subject to the regulator's approval. Telecom carriers see IPTV as new business opportunities intending to offer triple-play services, which include fixed phone lines, ADSL and access to a TV bouquet, all under one subscription. They want to deliver video over their own IP infrastructure as a way to grow average revenue per user (ARPU) in the face of pressures on their core revenues, which are declining for traditional circuit-switched voice services. IPTV services give carriers a strong edge to play in response to the competitive threat from cable operators. Based on the same protocols as the internet, IPTV gives carriers a strong hand to play. In addition to offering traditional cable TV-like services, the IPTV platform gives carriers an open platform for delivering a wide range of advanced entertainment services. This has the dual benefit of responding to the cable threat with a superior offering while also opening new revenue opportunities not available on cable TV platforms.

Korea's topography provides DSL a significant advantage over cable broadband in data delivery, so telcos enjoy a huge market share because they can deliver higher data speeds to the home. Current single-channel cable modem technology is too outdated to assist cable operators on this front. Therefore, cable operators see the two IPTV operators as dominant carriers who will swamp subscribers (Shim, 2005b). Korea's cable industry has been suffering from low market penetration. Pay cable television has not proven highly popular in Korea despite the limited number of terrestrial-based channels and the lack of satellite television. Only 15 percent of homes subscribed at the end of 2004. One reason is that relatively inexpensive alternatives such as videotapes and Digital Video Discs are widely available. In addition, a recent enforcement of channel tiering system has been an adverse factor to cable operators. The channel tiering system allows subscribers to select their favorite channels, the monthly charges are directly fed back to channel agents, and channel agents are devoted to 
the upgrade of program qualities. To make matters worse for cable operators, they also face challenges not only from IPTV, but also digital satellite broadcasting. Cable subscribers have complained about schedules dominated by re-runs, causing thousands to migrate to satellite broadcasting, Skylife, with its wealth of content. Therefore, video-on-demand or any content on demand through IPTV is a complete substitute for pay cable television. Once telecom carriers are offering the bundled IPTV services, cable operators have little strategic edge to capture customers. Cable operators have scrambled the signals from telco to prevent telecom entities from entering the broadband-based TV service, calling it the realm of broadcasters and not telecom players. Telecom carriers counter-ague that they are losing subscribers (85,000 since VolP service) to cables' IP-based phone service and are anxious to grab a share of cables' pay TV market. Telecom carriers criticize that cable operators are pioneering new business areas of internet telephony (VolP), which is telco's domain, but cable operators are opposing telecom carriers providing IPTV. In addition, cable operators are using franchising process as a barrier to cable entry, which has been used to insulate itself from competition. The two telecom carriers hope to convince regulatory bodies that its product is all-together different from traditional cable and, as such, should be regulated differently from the traditional cable television industry.

Industrial experts have requested the government should ease its anti-monopoly regulations on cable service operators to help them focus on investment in content and survive in what they describe as a war with capital. Reflecting this request, recently MIC announced the tentative ban on telcos from providing content over IP. The MIC's definition of IPTV as "Internet Content on Demand" favors cable operators at least for the time being.

\section{Broadcasters vs telcos}

Along with cable operators, three national broadcasters in Korea are opposing telecommunications carriers providing IPTV. The troubling issue of content retransmission in the previous DMB has been occurring again in the IPTV development. Related to content, the fundamental question in the IPTV has been the retransmission of over-the-air content via internet protocols. Three national broadcasters have opposed to share their content with the IPTV providers worrying that they might lose their control on content. Content providers in IPTV include three broadcasting carriers and a group of application and content providers. The key issue in the content layer is a retransmission of broadcasting content over IPTV. The three national terrestrial broadcasting carriers intend to maximize their power, leveraging the content retransmission issue. Their argument of opposing their content to be used in IPTV is that broadcasting content cannot be commercialized in the name of public interests. However, the real motivation is that they do not want to lose their hegemony in the market where they had been protected under public broadcasting systems.

The broadcasters argue that broadcasting content should not be commercialized in the name of public interests. Telecom carriers counter-argue that subscription-based content is the only way to operate IPTV in a sustainable business model. The view of broadcasters seems to remain in the vertical silo perspective. This discrepant view implies the significant disjunction in the socio-technical perspective. $\mathrm{KBC}$ has been unable to issue a business license needed for the IPTV in the face of strong protest from some of its member broadcasters. The protesters' argument is that retransmission would tarnish the public interest of broadcasting. The nation's main broadcasters refuse to air their programs through the IPTV services. The three major terrestrial broadcasters estimated that the competitiveness of the IPTV content would drop if the retransmission of programs via the IPTV service is permitted. The retransmission issue is a problem that should be settled through negotiations among the providers.

Telecommunications carriers have been seeking a way to retransmit over-the-air broadcasting content to IPTV. Mobile telecom industries have been suffering from freezing revenue in the saturated wireless market. Mobile industries now turn their strategic attention to a new cash cow of IPTV. The thorny issue around content retransmission reveals the implication of a layer approach in IPTV. The broadcasting carriers still have a vertical model of communication, that is, broadcasting infrastructure offers broadcasting content, 
whereas telecom infrastructure is provisioned to provide telecom content, and internet infrastructure offers internet content.

\section{Conclusion}

This study, drawn from extensive industry research and interviews with IPTV insiders and regulators, provided the background and dynamics on the development of IPTV, including technology innovation, industrial change, operators' strategies, consumer demand factors, and regulatory influences. It also collected survey data from prospective users to investigate the users' attitudes and perceptions on IPTV. It assessed the technical feasibility, profiled market and user, and surveyed the IPTV regulatory environment including profiles of key regulatory agencies and summarization of current issues. It profiled key industry players including service providers, industry consortia, content providers and handset vendors. Finally, it summarized the interaction of these factors in order to see the dynamics of IPTV development.

Socio-technical analysis implies that the emergence of IPTV will likely continue to be a socially and technologically complex development. Socially, IPTV will involve many firms, structures, and legal entities, industries, and user groups. Technologically, IPTV will accelerate cognate technologies of infrastructure, applications, and services evolving toward next generation network environment. The analysis further reveals a socio-technical disjuncture in the development of IPTV between the technical, political, and industrial change. It also highlights the tensions between and among the different regulators and various industries. The development of IPTV in Korea is stuck at the intersection of engineering and social discourses, because the advancement of IPTV technologies poses to debilitate current regulatory structure, destruct current market and gradually introduce new structure of market, industry, and regulation, that is, a layered model of next generation network.

The quantitative survey results reveal the importance of content and service in IPTV development, which implies that IPTV is a "sociotechnical ensemble" (Pinch and Bijker, 1984, p. 274) rather than a simple technical tool or electronic appliance. The development and analysis of IPTV should not be approached purely from social and not wholly from technical perspective; IPTV is a socio-technical ensemble, which means that we should be able to trace the social construction of IPTV and we should be able to trace the relations that go into stabilizing each stakeholder. Tracing relation is what Pinch and Bijker leave as an open question. They argue for a need to produce "other differentiations between dependent and independent variable, between foreground and background - differentiations that are not based on the distinction between the social and the technical' (Pinch and Bijker, 1984, p. 274). Rather than talk of the social or technical, this paper addresses the particular relations around the IPTV development.

At the planning stage of the IPTV product, there are concerns in the industry over whether there will be sufficient actual demand for the IPTV services. These concerns came from the industry's experience on IMT-2000, ISDN, dot.com bubble and, in particular, DMB, which had focused mainly on the supply side and some services were not used as anticipated (Shin et al., 2006). Stakeholders now believed from the DMB experience that the IPTV's success depended on the creation of application services that would be highly demanded and widely used. The public must be prepared to use the new technologies and services so that they can benefit from the enhanced capabilities. The government pursued a variety of policy measures for creating IPTV demands as well as networks.

The web of stakeholder analysis implies that the further development of IPTV in Korea is likely to comprise multiple stages, predicated by several significant events: the deployment of a broadband convergence network and FTTH, the resolving of content issues, establishing standards, and the introduction of new regulatory frameworks. Considering the multiple stages, IPTV may not be successful as a stand-alone technical artifact. Rather, it will be viable as part of a bundle of different services, which may involve high-speed internet connections, dual mode telephony, and perhaps mobile TV. This nature of IPTV sheds light on the socio-technical challenges that IPTV will face. 
High attention has been paid to IPTV because of its possible impact on the current technology environment and potential catalytic power to the next generation networks. Unlike the over-hyped forecasts, however, the reality and prospectus are not optimistic enough to expect full success of IPTV. The absence of strategic integration among policy, technology, and industry, the prospectus of IPTV somewhat over-evaluated with a self-fulfilling prophesy thus the concerns of latent disruption and uncertainty are largely sidelined. Many hyped expectations and optimistic projections - such as when to succeed, what impact, and how much economic development - are all dependent on the dynamics of the socio-technical system components and interplays of web of stakeholders. We can predict a more precise IPTV trajectory as a product of socio-technical ensemble if we can see IPTV within the domain of the co-evolving environments of technology, market, regulation and industry.

Finally, both web of stakeholder and socio-technical analysis show that different stakeholders have different notions towards IPTV: users groups perceive IPTV as an advanced appliance; telcos see IPTV as a complete system (end-to-end system), manufacturers (LG Electronics and Samsung Electronics) think of IPTV as equipment; cable operators and ISPs see IPTV as a network access to transport their content and services, and,: broadcasters see IPTV as an extension or a variation of broadcasting. This conceptual discrepancy is nicely fit to the notion of Interpretive Flexibility by the Relevant Social Groups of Bijker (1995). The paper closes by calling for further research tracing the actual diffusion of IPTV with a focus on the conceptual discrepancy among stakeholders identified in this study.

\section{References}

Barton, S., Hogan, N. and Lambert, E. (2002), "Multiple-methods and practical research: the value of using both quantitative and qualitative data to assess a cognitive based program", paper presented at the 2002 American Society of Criminology Conference, Philadelphia, PA, November.

Bijker, W.E. (1995), Of Bicycles, Bakelites and Bulbs: Towards a Theory of Sociotechnical Change, The MIT Press, Cambridge, MA.

Choudrie, J. and Lee, H. (2003), "A web of stakeholders and strategies: a case of broadband diffusion in South Korea"', Journal of Information Technology, Vol. 18, December, pp. 281-90.

Damsgaard, J. (1996), "The diffusion of electronic data interchange: an institutional and organizational analysis of alternative diffusion patterns", PhD thesis, Department of Computer Science, Aalborg University, Aalborg.

Freeman, R.E. (1984), Strategic Management: A Stakeholder Approach, Ballinger, Cambridge, MA.

Han, G. (2003), "Broadband adoption in the United States and Korea: business driven rational model versus culture sensitive policy model", Trends in Communication, Vol. 11 No. 1, pp. 3-25.

Khanna, T. and Palepu, K. (1999), "The right way to restructure conglomerates in emerging market", Harvard Business Review, Vol. 77 No. 4, July-August, pp. 125-34.

Kim, C. (2001), "Korea information infrastructure and broadband service", paper presented at OECD Meeting, Paris, June 12.

King, J. (1994), "Institutional factors in information technology innovation", Information Systems Research, Vol. 5 No. 2, pp. 139-69.

MacKenzie, D. and Wajcman, J. (Eds) (1999), The Social Shaping of Technology, 2nd ed., Open University Press, Philadelphia, PA and Milton Keynes.

Menard, S. (1995), Applied Logistic Regression Analysis, Sage, Thousand Oaks, CA.

Mitroff, I. (1983), Stakeholders of the Organisational Mind: Toward a New View of Organisational Policy Making, Jossey-Bass, London.

OECD (2004), "OECD information technology outlook", available at: www.oecd.org/sti/ito (accessed October 2005).

Orlikowski, W.U. (2002), "Knowing in practice: enacting a collective capability in distributed organizing", Organization Science, Vol. 13 No. 3, May-June, pp. 249-73. 
Papazafeiropoulou, A. and Pouloudi, A. (2000), "The government's role in improving electronic commerce adoption", Proceedings of the Eighth European Conference on Information Systems (ECIS 2000), Vienna, Austria, 3-5 July.

Pinch, T.J. and Bijker, W.E. (1984), "The social construction of facts and artifacts: or how the sociology of science and the sociology of technology might benefit each other", Social Studies of Science, Vol. 14, pp. 399-442.

Sawyer, S. (2001), "Socio-technical structures in enterprise information systems implementation: Evidence from a five-year study", The 2001 IEEE EMS International Engineering Management Conference, IEEE Press, Albany, NY, October, pp. 172-8.

Sawyer, S., Allen, J. and Lee, H. (2003), "Broadband and mobile opportunities: a sociotechnical perspective", Journal of Information Technology, Vol. 18, pp. 121-36.

Shim, J. (2005a), "Korea's lead in mobile cellular and VolP phone service", Communications of the Association for Information Systems, Vol. 15, pp. 555-66.

Shim, S. (2005b), "South Korean cable TV firms face challenges from digital media", iCable News, No. 38, February 28.

Shin, D. (2004), "Technology convergence and regulatory challenge: a case from Korean digital media broadcasting", info, Vol. 7 No. 3, pp. 47-58.

Shin, D., Kim, W. and Lee, D. (2006), "A web of stakeholders and strategies in the development of digital multimedia broadcasting", The International Journal on Media Management, Vol. 8 No. 2, pp. 70-83.

Williams, R. and Edge, D. (1996), "The social shaping of technology", Research Policy, Vol. 25, pp. 865-99.

Winner, L. (1997), "Artifacts/ideas and political culture", in Teich, A. (Ed.), Technology and the Future, St Martin's Press, New York, NY.

\section{Further reading}

Korea Broadcasting Commission (2004), "Korea Broadcasting Commission annual report", available at: www.kbc.or.kr/anuanlreport/2004

Korea Broadcasting Commission (2004), "Korea Broadcasting Commission bylaws", available at: www. kbc.or.kr/bylaws

Ministry of Information and Communication (2000), White Paper on Information and Communication, Ministry of Information and Communication, Jongno-gu (in Korean).

Papazafeiropoulou, A., Pouloudi, A. and Currie, W. (2001), "Applying the stakeholder concept to electronic commerce: extending previous research to guide government policy makers", Proceedings of the Hawaii International Conference on Systems Sciences (HICSS-34), Maui, Hawaii, 3-6 January.

Rosen, P. (1996), "Of artifacts, analysis, and alliteration: theory and politics in constructivist technology studies", Social Studies of Science, Vol. 26, pp. 705-11.

Sitter, L., Hertog, J. and Dankbaar, B. (1997), "From complex organizations with simple jobs to simple organizations with complex jobs", Human Relations, Vol. 50, pp. 497-534.

\section{About the author}

Dong $\mathrm{H}$. Shin has been Assistant Professor in the College of Information Sciences and Technology at Pennsylvania State University since 2004. He holds a PhD and a Master degree in Information Sciences and Technology from Syracuse University. Dong H. Shin can be contacted at: dxs75@psu.edu

To purchase reprints of this article please e-mail: reprints@emeraldinsight.com Or visit our web site for further details: www.emeraldinsight.com/reprints 